\title{
DESIGN CONSIDERATION OF A BOOSTER FOR TAIWAN PHOTON SOURCE
}

\author{
Gwo-Huei Luo, H.P. Chang, C.C. Kuo, K.K. Lin, H.J. Tsai and M.H. Wang \\ National Synchrotron Radiation Research Center, No. 101, Hsin-Ann Road, Hsinchu, Taiwan
}

\begin{abstract}
After more than 10 years' operation and expansion, the Taiwan Light Source (TLS) of National Synchrotron Radiation Research Center (NSRRC) reaches very stable operation condition. The storage ring has better than $96 \%$ of beam availability annually. There are 6 Insertion Devices (IDs) in a six-folds symmetric machine and three superconducting wigglers are going to be installed in achromatic sections. Two superconducting IDs and one Superconducting RF (SRF) cavity were installed in recent year, which intended to push the photon energy to hard $\mathrm{x}$ ray regime and double the photon flux with better beam quality. Beamlines and experimental stations occupied all over the experimental area. The uproar for more beamlines in higher photon energy with higher brightness was frequently transpired from users' community. The NSRRC proposed a new design and construction timetable for a median-energy light source, Taiwan Photon Source (TPS). This paper will present two draft designs of booster and the considerations of the design criteria for new booster to work with a very lowemittance and top-up injected storage ring.
\end{abstract}

\section{INTRODUCTION}

The Taiwan Light Source provides $200 \mathrm{~mA}, 1.5 \mathrm{GeV}$ electron beam to generate photons source for academic and industrial research scientists. The storage ring is a six-fold symmetric Triple-Bend-Archomat (TBA) lattice with six straight sections. The strong demanding of synchrotron light in $\mathrm{x}$-ray regime made the accelerator physicists try the efforts to squeeze the space at injection and RF sections to accommodate super-conducting highfield insertion devices.

It was also a strong demand to increase the photon flux and reduce the photon fluctuation. A SRF cavity was installed to replace two Doris cavities. The SRF cavity was designed to be a higher-order-modes free cavity and had the capability to provide $8 \mathrm{MV} / \mathrm{m}$ accelerating gradient with power handling capability up to $200 \mathrm{~kW}$.

The demanding of lower emittance, small gap insertion device and doubling the stored beam current made us to evaluate the feasibility of top-up operation [1] at TLS and designing a new storage ring with higher energy for users. A $3.0 \mathrm{GeV}$ storage ring with circumference of $518.4 \mathrm{~m}$ and very low emittance was studied [2]. The new storage ring can be nicely accommodated with current infrastructure of NSRRC.

Here we describe two booster layouts that have been considered for the TPS. They are based on FODO lattice with missing magnets to match the dispersion at the straight section. At this stage, it is difficult to describe which one will be the best alternative. However, it suffices to say that either option will fulfil requirements.

\section{BOOSTER SHARING SAME TUNNEL WITH STORAGE RING}

The pre-injector, electron gun and Linac as shown in Fig. 1, will be a commercial turnkey system. The electrons through LINAC should deliver at least $100 \mathrm{MeV}$ beam energy for subsequent injection into booster.
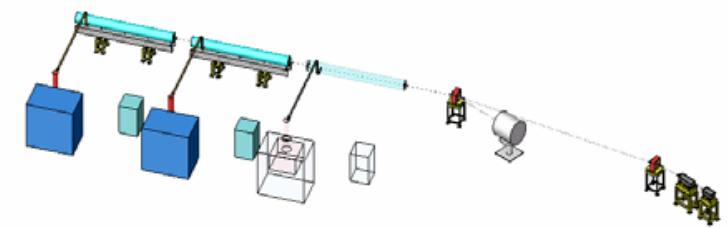

Figure 1: Schematic layout of proposed $100 \mathrm{MeV}$ Linac injector.

The FODO lattice is the most commonly used and best understood lattice for booster synchrotron. Very low beam emittances can be achieved by using very short cells. For an isomagnetic FODO lattice, the beam emittance is given by [3]

$$
\varepsilon_{\text {FODO }}=C_{q} \gamma^{2} \theta^{3} \frac{I_{b}}{I_{b 0}} \frac{\langle H\rangle}{\rho \theta^{3}} .
$$

In the case of $<\mathrm{H}>/\left(\rho \theta^{3}\right) \approx 1.25$, minimum beam emittance in practical units is

$$
\varepsilon_{\text {FODO }}(m-r a d)=97.53 * 10^{-13} \frac{I_{b}}{I_{b 0}} E^{2}[\mathrm{GeV}] \theta^{3}[\mathrm{deg}]
$$

where $I_{b 0}$ is the actual effective length of one bending magnet and $2 I_{b}$ is the length of a FODO cell.

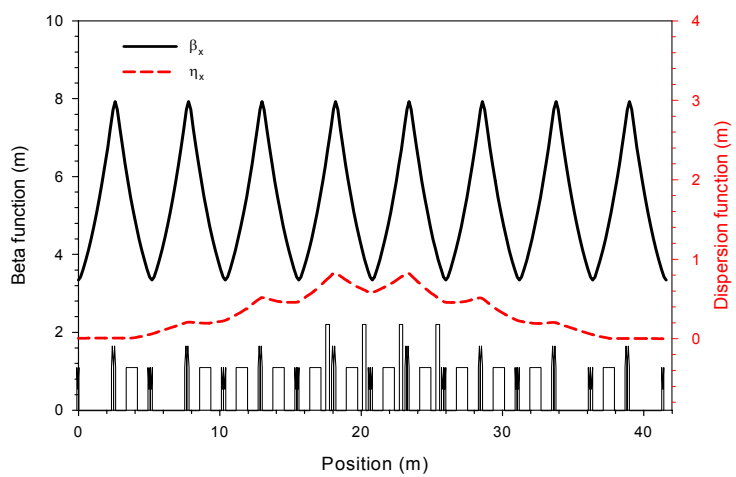

Figure 2: Optical function for a proposed high-emittance booster, sharing the same shielding tunnel with the storage ring. 
The lattice structure of booster will be based on the FODO cell. It is desired to have the dispersion function vanish or at least be small in magnet free straight sections to simplify injection and avoid possible instabilities if RF cavities a placed. The magnetic lattice of this booster option is a separated function FODO lattice in the preliminary design stage. Chromaticity correction for booster was also carefully analysed and developed.

The fundamental parameters of a high-emittance booster ring, sharing the same shielding tunnel with storage ring, is shown in Table 1 . The lattice is a 12 super-period structure to have easier arrangement of injection and extraction schemes and better transverse separation with storage ring. The optical functions, $\beta_{\mathrm{x}}$ and $\eta_{\mathrm{x}}$, in one super-period are shown in Fig. 2. On-energy and off-energy tracking of dynamic aperture, by BETA code [4], is shown in Fig. 3 with large enough dynamic aperture for easy operation.

Using the same dipole and quadrupole magnets' layout, a set of parameters with low emittance lattice was found as shown in Table 1. The real space dynamic aperture in $\mathrm{x}$-plane will shrink to $\pm 12 \mathrm{~mm}$ with the correction of chromaticity to zero. A more detail study will be needed for this low emittance lattice.

The evolution of emittance and energy spread for highemittance and low-emittance lattices was carried out as described in Section 4. It shows the ramping cycle for a booster, sharing the same tunnel, will be limited to $2.5 \mathrm{~Hz}$.

Table 1. Beam parameters for the high emittance lattice

\begin{tabular}{|l|r|r|}
\hline Parameters & High emittance & Low emittance \\
\hline Extraction energy $[\mathrm{GeV}]$ & 3 & 3 \\
\hline Injection energy $[\mathrm{GeV}]$ & 0.1 & 0.1 \\
\hline Circumference [m] & 499.2 & 499.2 \\
\hline Revolution time [s/turn] & $1.665^{*} 10^{-6}$ & $1.665^{*} 10^{-6}$ \\
\hline Tune $v_{\mathrm{x}} / v_{\mathrm{y}}$ & $16.13 / 7.18$ & $27.11 / 13.16$ \\
\hline Nature chromaticity $\left(\xi_{\mathrm{x}} / \xi_{\mathrm{y}}\right)$ & $-14.12 /-13.96$ & $-30.5 /-22.8$ \\
\hline Emittance [nm-rad.] & 36.6 & 5.30 \\
\hline $\begin{array}{l}\text { Damping time }\left(\tau_{\mathrm{x}} / \tau_{\mathrm{y}} / \tau_{\mathrm{e}}\right) \\
\text { [ms] }\end{array}$ & $26.1 / 25.6 / 12.6$ & $25.8 / 25.6 / 12.7$ \\
\hline Damping partition $\left(\mathrm{J}_{\mathrm{x}} / \mathrm{J}_{\mathrm{y}} / \mathrm{J}_{\mathrm{e}}\right)$ & $0.98 / 1.0 / 2.02$ & $0.993 / 1.0 / 2.01$ \\
\hline Momentum compaction $\alpha$ & 0.00492 & $1.68^{*} 10^{-3}$ \\
\hline Radiation loss $[\mathrm{keV} /$ turn] & 390.8 & 390.8 \\
\hline Energy spread & $5.97 * 10^{-4}$ & $5.99^{*} 10^{-4}$ \\
\hline Quantum lifetime $[\mathrm{minutes}]$ & $13.6(@ 0.7 \mathrm{MV})$ & $2.02 * 10^{20}(@ 0.8$ \\
& & $\mathrm{MV})$ \\
\hline
\end{tabular}

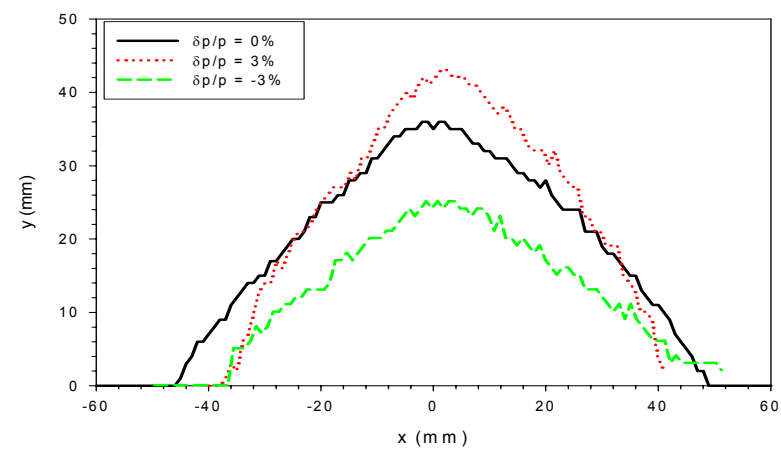

Figure 3: Dynamic aperture tracking for the cases of onand off-energy, $\pm 3 \%$.

\section{A SEPARATED BOOSTER RING}

The fundamental parameters of a separated booster synchrotron are shown in Table 2 . It is desired to evaluate the beneficial of a booster sharing the tunnel with storage ring and a separated booster before the chosen of which type of booster being built. The optical function of the designed booster is shown in Fig. 4. It is a two-fold symmetric lattice with one straight section for injection and RF cavity and one straight section for extraction. Figure 5 indicates that a multi-cells cavity is necessary to have adequate quantum lifetime for the extraction energy at $3.3 \mathrm{GeV}$.

Table 2. Beam parameters for a separated booster

\begin{tabular}{|l|r|}
\hline Extraction energy $[\mathrm{GeV}]$ & 3 \\
\hline Injection energy $[\mathrm{GeV}]$ & 0.1 \\
\hline Circumference $[\mathrm{m}]$ & 162 \\
\hline Revolution time $[\mathrm{s} /$ turn] & $0.5404 * 10^{-6}$ \\
\hline Tune $v_{\mathrm{x}} / v_{\mathrm{y}}$ & $7.11 / 4.17$ \\
\hline Nature chromaticity $\left(\xi_{\mathrm{x}} / \xi_{\mathrm{y}}\right)$ & $-8.02 /-5.97$ \\
\hline Emittance $@ 3 \mathrm{GeV}[\mathrm{nm}-\mathrm{rad}]$. & 126 \\
\hline Damping time $\left(\tau_{\mathrm{x}} / \tau_{\mathrm{y}} / \tau_{\mathrm{e}}\right)[\mathrm{ms}]$ & $6.06 / 5.76 / 2.81$ \\
\hline Damping partition $\left(\mathrm{J}_{\mathrm{x}} / \mathrm{J}_{\mathrm{y}} / \mathrm{J}_{\mathrm{e}}\right)$ & $0.95 / 1.0 / 2.05$ \\
\hline Momentum compaction $\alpha$ & 0.025 \\
\hline Radiation loss $[\mathrm{keV} / \mathrm{turn}]$ & 562.78 \\
\hline Energy spread & $7.11 * 10^{-4}$ \\
\hline RF acceptance & $3.78^{*} 10^{-3}(@ 1.2 \mathrm{MV})$ \\
\hline Quantum lifetime $[\mathrm{minutes}]$ & $2.29(@ 1.2 \mathrm{MV})$ \\
\hline
\end{tabular}

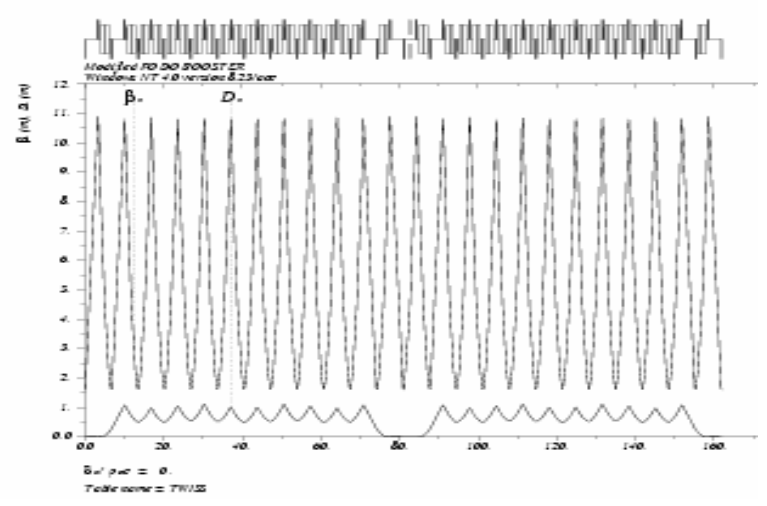

Figure 4: Optical function, $\beta \mathrm{x}$ and $\mathrm{Dx}$, for a separated booster ring.

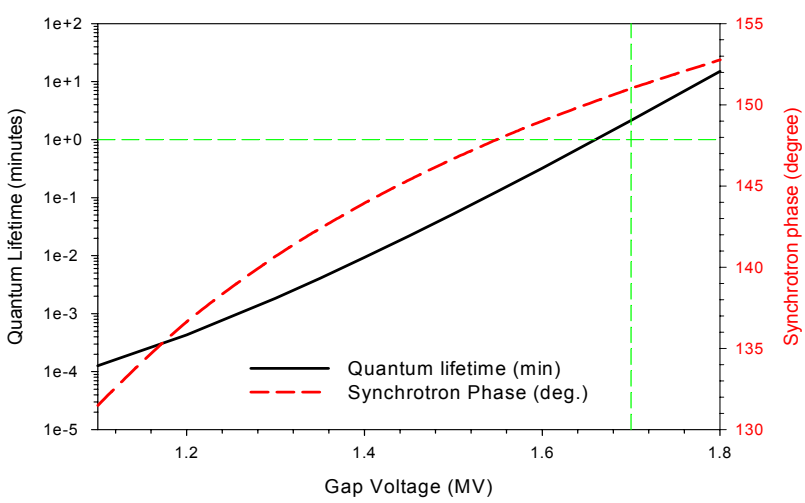

Figure 5: Quantum lifetime for a separated booster ring. The target extraction energy is $3.3 \mathrm{GeV}$. 
Figure 6 presents the dynamic aperture tracking for the cases of on- and off-energy particles for the separated booster ring. Using the method discussed in Section 4, the ramping cycle can be as high as $5 \mathrm{~Hz}$.

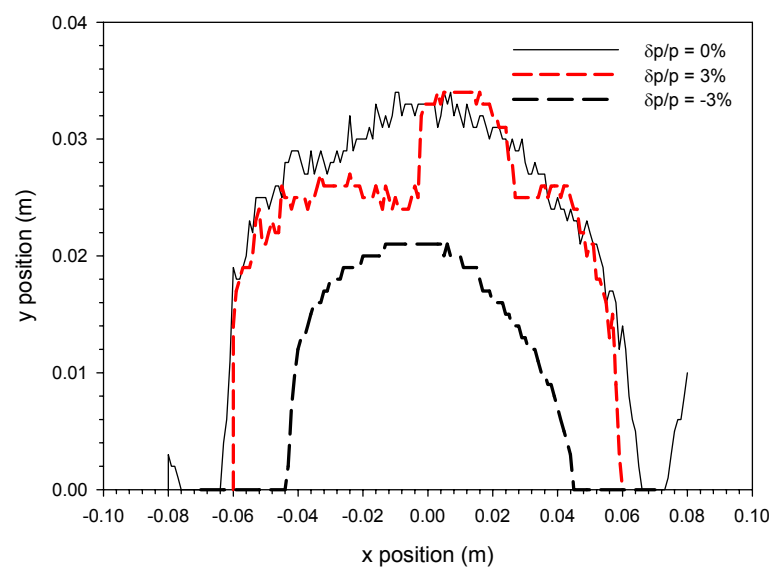

Figure 6: Dynamic aperture tracking for a separated booster ring.

\section{EVOLUTION OF EMITTANCE AND ENERGY SPREAD}

Table 1 and 2 give the fundamental parameters for a booster ring with high-emittance, low-emittance lattices and a separated booster ring respectively. During the acceleration period, the emittance and energy spread are time-dependent dynamic parameters. The quantum excitation, adiabatic damping and radiation damping lead the elections to be a competition process changing the dynamic parameters as function of time.

During the acceleration, the transverse emittance as function of energy is given as $[5,6]$,

$$
\frac{1}{\varepsilon} \frac{d \varepsilon}{d t}=-\left(\frac{1}{\gamma} \frac{d \gamma}{d t}+\frac{2}{\tau_{x}}\right)+\frac{Q_{x}}{2 \varepsilon},
$$

where $\varepsilon, \tau_{x}$, and $Q_{x}$ are function of $\gamma$ and $\gamma$ is the relativistic energy of the electron. The $\tau_{x}$ represents the damping time at energy of $\gamma$ and $Q_{x}$ stands for the energy fluctuation due to synchrotron radiation emission. In a similar fashion, we can also write done the evolution of energy spread during acceleration.

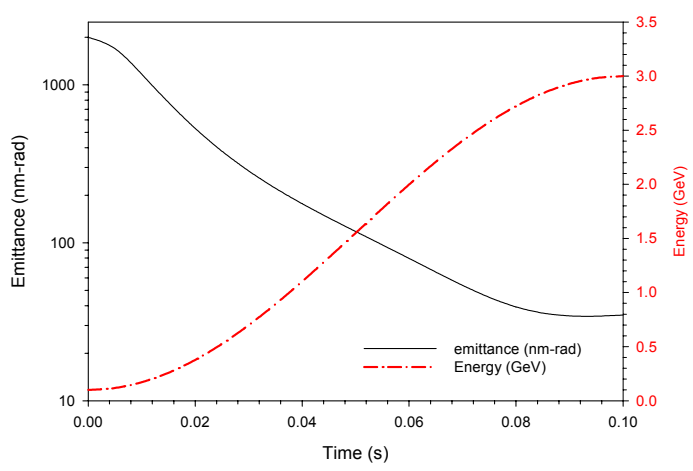

Figure 7: Evolution of the emittance during energy ramping by using sinusoid ramping curve.
The evolution of beam emittance, using the parameters listed in Table 1, is shown in Fig. 7. We assume the ramping curve is sinusoid with $5 \mathrm{~Hz}$ of cycling frequency. The target extraction emittance is achievable. The evolution of energy spread is shown in Fig. 8 using the same parameters set as the case of Fig. 7. The fastest ramping frequency is $2.5 \mathrm{~Hz}$ to reach the target extraction energy spread. Hence, the cycling frequency of a booster ring, sharing the same tunnel with storage ring, is $2.5 \mathrm{~Hz}$ to have all dynamic parameters reaching design value. The same evaluation process is adapted to calculate the cycling frequency for various designs.

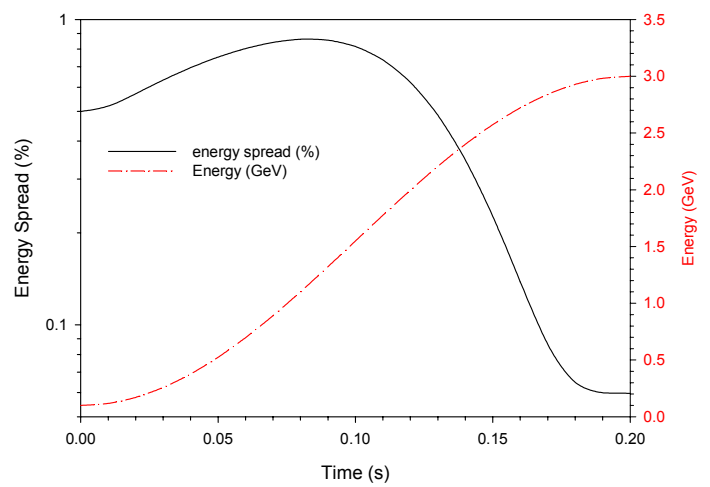

Figure 8: Evolution of energy spread during energy ramping by using sinusoid ramping curve.

\section{SUMMARY}

The circumference of booster has been adjusted to sharing the same tunnel with the storage ring. Two latticesettings, with same physical layout, were investigated. From the calculation, we adopted cycling frequency of $2.5 \mathrm{~Hz}$ in order to allow fully damped emittance and energy spread. The re-utilizing of two Doris cavities can provide sufficient accelerating gap voltage for the layout. Injection and extraction scheme of the boosters need to be designed. FODO lattice constructed with combinedfunction dipole will be an interesting design option.

A separated booster ring to fulfil the energy ramping is studied. The compact booster size can be built separately without interference with the construction of storage ring and potentially contracted to single vendor as a turnkey system. It will require a new cavity to provide necessary acceleration.

\section{REFERENCES}

[1] G.H. Luo, et al., "The Status of Topping Up Injection at SRRC, "APAC'04, 2004

[2] C.C. Kuo et al., "Lattice Study for the Taiwan Photon Source," PAC 2005, this proceeding.

[3] H. Wiedemann, Particle Accelerator Physics, Springer-Verlag, Berlin Heidelberg, 1998.

[4] J. Payet et al, BETA, LNS version.

[5] M. Sands, The Physics of Electron Storage Rings, SLAC-121, 1970

[6] K.K. Lin and K.C. Cheng, "Ramping Behavior of Booster Synchrotron,” Nucl. Sci. J. 25, 1988. 